\title{
Vibrations in amorphous silica
}

\author{
Bishal Bhattarai ${ }^{\mathrm{a}}$, D. A. Drabold ${ }^{\mathrm{a}}$ \\ ${ }^{a}$ Department of Physics and Astronomy, Ohio University, Athens. Ohio 45701
}

\begin{abstract}
In this paper, we describe the vibrational properties of a realistic 648-atom model of $\mathrm{SiO}_{2}$ [1]. The normal modes of the model are computed with the ab initio density functional code SIESTA [2]. The static structure factor and the electronic density of states are in reasonable agreement with the experimental data. The vibrational density of states is analyzed by evaluating the partial density of states and localization. The vibrational modes were further investigated by calculating phase quotient and stretching character, and constructing animations of specific nodes of interest. We have obtained a low temperature specific heat in qualitative agreement with experiments, including a feature resembling the Boson peak, and the modes that give rise to this feature.
\end{abstract}

Keywords: VDOS, sillica, decorate and relax, specific heat

\section{Introduction}

Amorphous $\mathrm{SiO}_{2}$ is probably the most researched amorphous material. Neutron scattering[3],[4],[5] and Raman spectroscopy[4],[6], along with infrared spectroscopy[7] are employed to understand the vibrational and structural properties[3] of a-SiO 2 . Also, several computer models of the materials exist. The models are prepared using empirical potentials, ab initio or reverse monte-carlo techniques[8]. In this paper, we characterize an ab initio "decorate and relax "[9] model of a$\mathrm{SiO}_{2}$ and present extensive new results on its phonons.

While there has been much theoretical research to understand the physics of a-SiO $\mathrm{O}_{2}$, a perfect model for the vibrational properties and derived quantities like the vibrational specific heat has been lacking. Phonon calculations demand a precise calculation to satisfactorily model the vibrational excitations. Significant controversy exists in the literature about artifacts of small structural models and/or imperfect interatomic potentials. We should note too that there is some nonnegligible variance in experimental data on the vibrational density of states, as we describe later.

We have employed a 648-atom model of a- $\mathrm{SiO}_{2}$ with self-consistent field density functional interactions to study the vibrational properties of the system. We study the vibrational properties by diagonalizing the dynam-

Email addresses: bb248213@ohio .edu (Bishal Bhattarai), drabold@ohio.edu (D. A. Drabold)

Preprint submitted to Journal of Non-Crystalline Solids ical matrix which gives us the eigenvalues and eigenvectors (classical normal modes). Our results are in improved agreement with experiment and we present interesting new results for the low-temperature specific heat. We thoroughly characterize these modes, by including the Taraskin-Elliott correction (connecting experiment and theory), and computing phase quotients and estimating bond-stretching/bending behavior. Our paper is also of some interest as the largest (648-atom) ab initio study of vibrations in amorphous silica presented to date. Also, modes in complex systems like amorphous silica are often thought of very locally - as essentially molecular excitations, but of course this is a gross (albeit useful) simplification. This is shown clearly in animations that we provide as Supplementary Material for this paper. These animations emphasize the non-locality of the modes and, and are useful as a pedagogic aid.

The paper is organized as follows, In section II we discuss the simulation methods used. Section III provides a description of the structural properties of the model: the structure factor, pair correlation function and compare it with the known experimental results of the material. Section IV gives a brief description of the electronic properties of the model and the localization of the electronic states. The core of the paper is Section $\mathrm{V}$, which is devoted to the vibrational properties of the system: localization, identification of the vibrational states and the low-temperature specific heat. In Section VI, we summarize our findings.

February 2, 2016 


\section{Model}

The models of a-SiO 2 were obtained with the help of $a b$ initio code "Spanish Initiative for Electronic Simulations with Thousands of Atoms "(SIESTA) [2], a density functional code using the local-density approximation (LDA) with Ceperley-Alder (CA) exchange correlation functional. The 648-atom model of $\mathrm{g}-\mathrm{SiO}_{2}$ consisted of $216 \mathrm{Si}$ atoms and $432 \mathrm{O}$ atoms with a of cube side length of $22.3 \AA$. The simulation was performed with single- $\zeta$ basis, a self-consistent field and periodic boundary conditions, and the final model was relaxed using the conjugate gradient (CG) method. The simulation was initially performed at constant volume, sampling the Brillouin zone only the $\Gamma(\vec{k}=\overrightarrow{0})$ point to compute the forces and energies. During the final relaxation, the system was allowed to relax to zero pressure for the single- $\zeta$ Hamiltonian. This allowed the system volume to change and resulted in a non-cubic cell, with a density about $11.7 \%$ smaller than the experimentally reported density. ${ }^{1}$.

\section{Structural Properties}

In a neutron or X-ray scattering experiment, the static structure factor $S(Q)$ is measured. The Faber-Ziman definition of partial structure factor can be stated as [12],

$$
S_{\alpha \beta}(Q)=1+4 \pi \rho_{0} \int_{0}^{\infty} d r r^{2} \frac{\sin (Q r)}{Q r}\left(g_{\alpha \beta}(r)-1\right)
$$

where, $S_{\alpha \beta}(Q)$ is the partial structure factor and $g_{\alpha \beta}(r)$ is the partial pair correlation function or radial distribution function (RDF), which gives the corresponding structural information in real space. $S(Q)$, the total structure factor is obtained by summing the partial

\footnotetext{
${ }^{1}$ Previous simulations of this system have either constrained the volume to match experiment [10], or used empirical potentials that are concocted to reproduce the density[11]. This makes the pair correlations look slightly better, but then one is not considering vibrational excitations around the true minimum, an essential assumption of the Harmonic Approximation. In addition, by carrying out this zero-pressure simulation the cubic symmetry of the supercell also slightly breaks, yielding lattice vectors: $\langle 22.28,-0.24,-0.12\rangle$, $\langle-0.24,22.48,0.03\rangle$ and $\langle-0.13,0.02,22.07\rangle$. While the effect is fairly small compared to our earlier calculation (as characterized by the structure factor, and quantities like bond angle distributions), we think this is a more appropriate starting point than an artificially constrained case. We verified that with a more complete double- $\zeta$ basis, the lattice constant was indeed close to experiment, as expected. Since the vibrational calculations require $6 \mathrm{~N}$ force calls, a phonon calculation with the full basis would be quite expensive for a cell with 648 atoms
}

structure factor with an appropriate weighing of their concentration and neutron scattering length of the corresponding species.

$$
S(Q)=\sum_{\alpha \beta} c_{\alpha} b_{\alpha} c_{\beta} b_{\beta}\left[S_{\alpha \beta}(Q)-1\right]
$$

Conversely, the pair correlation function is[13],

$$
g(r)=1+\frac{1}{2 \pi^{2} \rho_{0}} \int_{0}^{\infty}[S(Q)-1] \frac{Q^{2} \sin (Q r)}{Q r} d Q
$$

\subsection{Structure factor and pair correlation function}

For investigating vibrational properties of materials, a good structural model is required and $a b$ initio interactions have been the most reliable. In Fig. 1 we have compared the static structure factor $S(Q)$ of the 648atom $\mathrm{SiO}_{2}$ model with neutron-diffraction experimental results [1]. The comparison of $S(Q)$ shows an excellent fit for most of the range of $\mathrm{Q}$, while there is some discrepancy after $Q \sim 11 \AA^{-1}$. This discrepancy is due to the density difference between model and experiment, and originating from finite size effects[1].

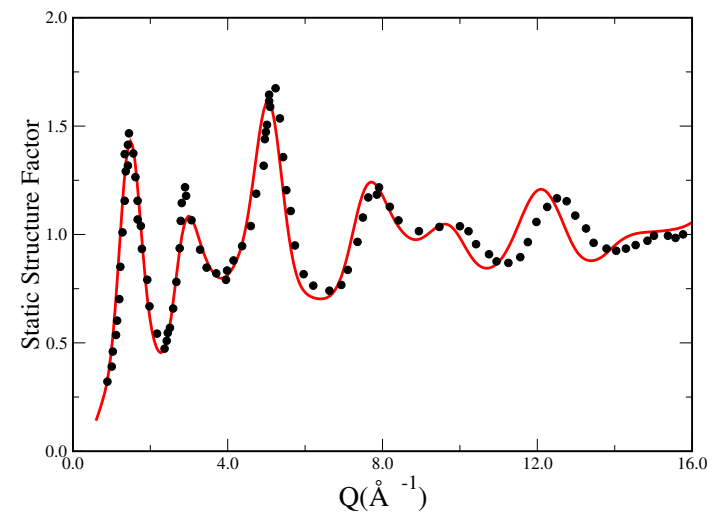

Figure 1: Calculated total neutron static structure factor for the 648atom $\mathrm{SiO}_{2}$ (solid red line) compared with experimental results[14] data (black circle, neutron diffraction)

Partial structure factors were also calculated for the 648-atom model, which gives us a better idea of the origin of various peaks in the total structure factor $S(Q)$. Results for the partial structure factor are given in Fig. 2. We can infer from Fig. 2 that the first peak in $S(Q)$ arises from all three partial factors. The second peak in $S(Q)$ arises mostly from the $S i-S i$ and $O-O$ correlations, while the contribution from $S i-O$ provides a partial cancellation. The third and fourth peak involves the contribution from $S i-S i, S i-O$ and $O-O$ depending upon the corresponding concentration and the 
scattering length. The counterpart of the structure factor (partial pair correlation) in real space is shown in Fig. 3. The first sharp peak of the pair correlation function gives us information about the bond length between nearest neighbors and the results obtained from the plot shows that the first peak of $S i-S i$ bonds is at $3.23 \AA$, similarly $S i-O$ and $O-O$ have their first maximum at $1.65 \AA$ and $2.71 \AA$ respectively. The average pair distances reported are, 3.100(0.113) $\AA$, 1.621(0.022) $\AA$ and $2.65(0.109) \AA$ for the $S i-S i, S i-O$ and $O-O$ respectively. The numbers in the brackets are the standard deviation for the data[15].

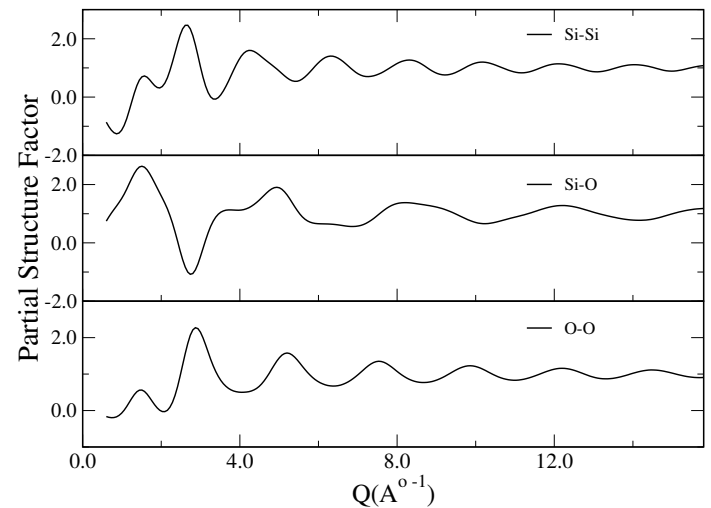

Figure 2: Calculated partial structure factors for the 648-atom model of $\mathrm{SiO}_{2}$

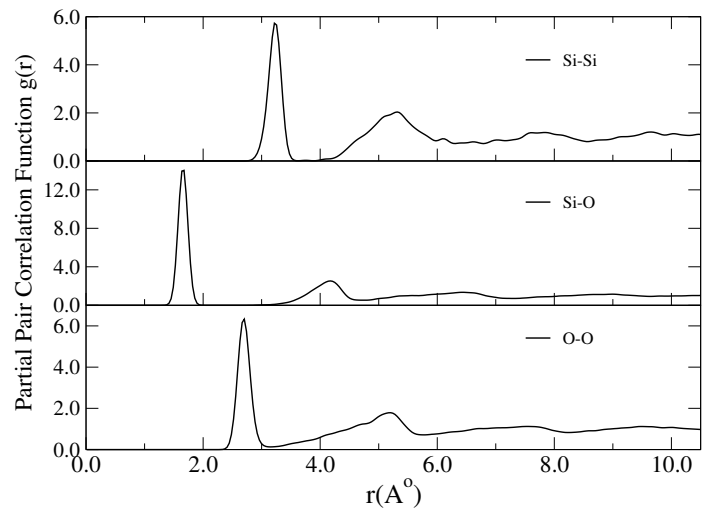

Figure 3: Calculated partial pair correlation functions for the 648atom model of $\mathrm{SiO}_{2}$

\subsection{Bond Angle distribution}

In Figs. 4, 5, we report the the bond angle distribution of our 648-atom model $\mathrm{SiO}_{2}$. This is in agreement with our previous results for the 192-atom model and 648-atom model[1]. The obtained structure has an average $O-S i-O$ angle of $108.97^{\circ}$ which is close to the tetrahedral angle of $109.47^{\circ}$. The broadening of the $S i-O-S i$ angles is consistent experimental results which also vary within the range of $142^{\circ}-152^{\circ}$ [16]. It is also to be noted that different measurements give slightly varying results. The FWHM (gaussian fit) for the two bond angle distributions $\mathrm{O}-\mathrm{Si}-\mathrm{O}, \mathrm{Si}-\mathrm{O}-\mathrm{Si}$ are $8.81^{\circ}$ and $31.21^{\circ}$ respectively.

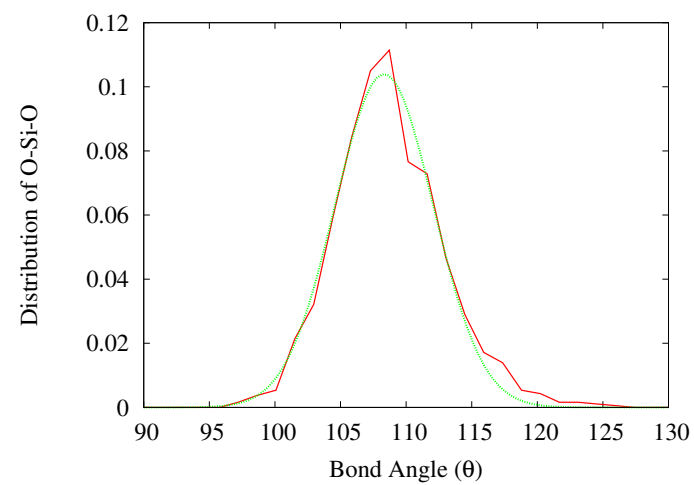

Figure 4: Bond-angle distribution function, $\mathrm{O}-\mathrm{Si}-\mathrm{O}$ (red),gaussianfit(dotted green)

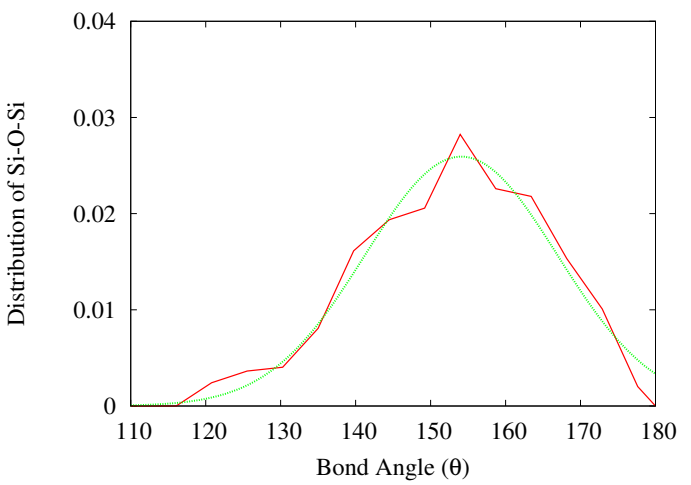

Figure 5: Bond-angle distribution function, $S i-O-S i$ (red,), gaussian-fit(dotted green).

\subsection{Ring statistics}

In Fig. 6 we have reported the ring statistics computed via ISSACS [17] for our sample of 648-atom $\mathrm{SiO}_{2}$. The ring statistics were obtained using King's shortest path [18].

From Fig. 6 we can see that there are no oddmembered rings, which shows the the model has no wrong (homopolar) bonds. Further, the maximum occurs for 12-member rings, which is in agreement with the previous finding for the structure rings of $\mathrm{SiO}_{2}$ [19]. 


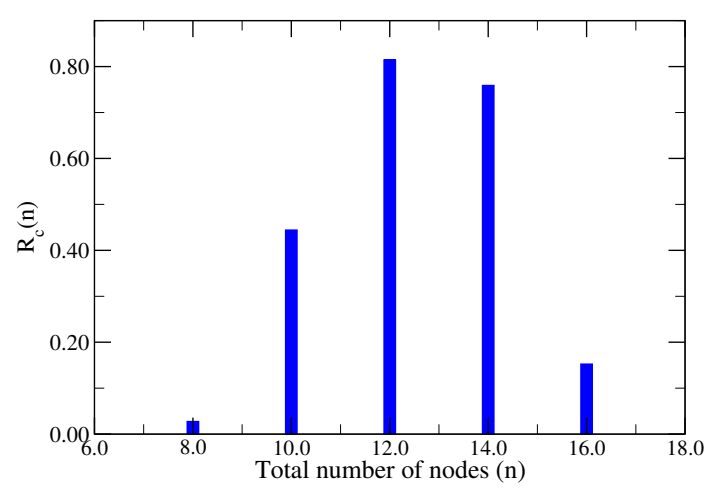

Figure 6: Number of Ring per Cell $\left(R_{c}\right)$ versus the number of $\operatorname{nodes}(\mathrm{n})$.

Our sample is devoid of 4- and 6-fold ring. Edgesharing tetrahedra is the origin of 4-fold rings and distortion for 6-fold rings[19].

\section{Electronic Properties}

The electronic properties were analyzed with the electronic density of states (EDOS) and localization of different energy eigenstates. The localization is obtained by calculating the inverse participation ratio (IPR), which is given by the relation[20],

$$
\mathcal{I}\left(\psi_{j}\right)=N \frac{\sum_{i=1}^{N} a^{j^{4}}}{\left(\sum_{i=1}^{N} a^{j^{2}}\right)^{2}}
$$

where, $\psi_{j}=\sum_{i=1}^{N} a_{i}^{j} \phi_{i}$ is the $j$ th eigenvector and $\phi_{i}$ is a local atom-centered basis orbital. Fig. 6 shows the plot of the EDOS and IPR. For a completely localized state, $\mathcal{I}\left(E_{i}\right)=1$ (only one atomic site contributing to that state). Small IPR are associated with extended states, in which case the electronic wavefunctions are more uniformly distributed in space.

\section{Dynamical Properties}

In this section, we discuss the classical normal modes in some detail. The dynamical matrix is the key ingredient to analyze the vibrational properties of the model, which is obtained from a well relaxed system for which the inter-atomic forces can be evaluated. The dynamical matrix is given by the relation[13],

$$
D_{\alpha i, \beta j}=\frac{1}{\left(m_{i} m_{j}\right)^{\frac{1}{2}}} \frac{\partial^{2} \phi}{\partial u_{\alpha i} \partial u_{\beta j}}
$$

where, $\phi$ is the total energy of the system, $m$ 's are the ionic masses of the atoms and $\alpha$ and $\beta$ are the Cartesian

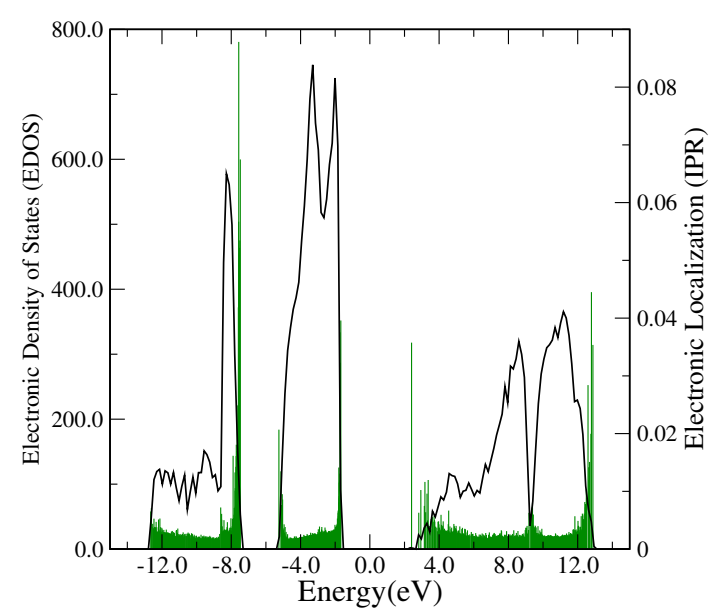

Figure 7: The Electronic Density of States (black,solid line) and the IPR (green,drop lines), with the Fermi Energy shifted to 0 ev.

coordinates of atom $i$ and $j$, respectively. The indices $i$ and $j$ run over the atoms. Now, when the $i^{\text {th }}$ atom is displaced by (a suitably small) $\Delta x_{\alpha i}$ in the $\alpha$ direction, the forces $\left(F_{\beta j}\right)$ on all the atoms are computed, so that the above relation can be replaced by[13],

$$
D_{\alpha i, \beta j} \approx \frac{1}{\left(m_{i} m_{j}\right)^{\frac{1}{2}}} \frac{F_{\beta j}}{\Delta x_{\alpha i}}
$$

For our system the dynamical matrix is obtained with the SIESTA[2] recipe by displacing each atom along the positive and negative Cartesian directions (in 6directions) with a magnitude of $0.02 A^{o}$. After, each such displacement an $a b$ initio force calculation was performed to obtain the force constant matrix resulting from the displacement of each atom from it's equilibrium position. The details of the constant force matrix calculation is given in [21]. The eigenvalue problem reads:

$$
\sum_{\alpha \beta} D_{\alpha i, \beta j} e_{\beta j}^{n}=-\omega_{n}^{2} e_{\alpha i}^{n}
$$

where, $\omega_{n}$ and $e_{\alpha i}^{n}$ represent the $3 N$ eigenfrequencies and the eigenvectors respectively.

If $\omega$ is the frequency then $g(\omega) d \omega$ describes the number of modes within the interval $[\omega, \omega+d \omega]$, where $g(\omega)$ represents the VDOS of the system. The VDOS for amorphous systems with $N$ number of atoms is defined as,

$$
g(\omega)=\frac{1}{3 N} \sum_{i=1}^{3 N} \delta\left(\omega-\omega_{i}\right)
$$


We evaluate the VDOS by Gaussian broadening the eigenvalue with the standard deviation $\sigma=2.5 \mathrm{meV}$ or $20.15 \mathrm{~cm}^{-1}$ : See Fig. 8. The first three frequencies are very close to zero, and arise from supercell translations. These have been neglected for the evaluation of both VDOS and derived quantities like specific heat.

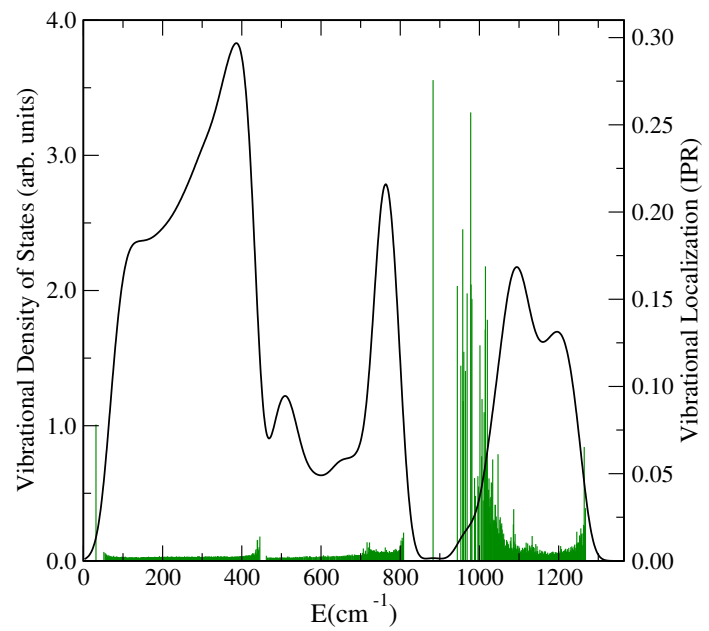

Figure 8: The Vibrational Density of States (black, solid line) and the IPR (green,drop lines). the highly localized modes near $1000 \mathrm{~cm}^{-1}$ are Si-O stretch modes.

\subsection{Assignment of normal modes}

The vibrational properties of amorphous $\mathrm{SiO}_{2}$ have been analyzed through various spectroscopies: neutron scattering[3],[4],[5], Raman scattering[3],[6] and infrared scattering[7]. The VDOS of amorphous silica shows several peaks. Our 648-atom model shows welldefined peaks occurring at low frequencies at 390 and $507 \mathrm{~cm}^{-1}$, and intermediate frequency at $766 \mathrm{~cm}^{-1}$. At high frequency the two peaks are at frequencies 1095 $\mathrm{cm}^{-1}$ and $1198 \mathrm{~cm}^{-1}$.

The VDOS of amorphous $\mathrm{SiO}_{2}$ has been well studied. In celebrated work, Bell and Dean [22] reported peaks at frequencies near $400 \mathrm{~cm}^{-1}, 550 \mathrm{~cm}^{-1}, 750 \mathrm{~cm}^{-1}$ and $1050 \mathrm{~cm}^{-1}$. Bell and Dean [22] were able to evaluate the effects of vibration due to the non-bridging oxygen atoms and their effects on VDOS. The importance of the motion of non-bridging oxygen atoms was vital for the peaks obtained at $300 \mathrm{~cm}^{-1}$ and $850 \mathrm{~cm}^{-1}$. The bending motion of non-bridging oxygen (NB) plays a large part for motion at $300 \mathrm{~cm}^{-1}$ and the peaks observed at $850 \mathrm{~cm}^{-1}$ is mostly due to the stretching motion of non-bridging oxygen (NS).

\subsection{Species projected VDOS}

Below, we present the atomic species-projected VDOS along with the total VDOS. The total density of states is projected onto the two species according to their respective weights of the eigenmodes and they satisfy the relation $g(\omega)=\sum_{\alpha} g_{\alpha}(\omega)$. The partial density of states can be defined as[10],

$$
g_{\alpha}(\omega)=\frac{1}{3 N} \sum_{i=1}^{N_{\alpha}} \sum_{n}\left|e_{i}^{n}\right|^{2} \delta\left(\omega-\omega_{n}\right)
$$

The vibration among the various species is mostly dominated by the oxygen atoms. From Fig. 9 we can infer that the vibrational motion of both the atoms $\mathrm{Si}$ and $\mathrm{O}$ are mixed through the spectrum except near 766 $\mathrm{cm}^{-1}$, where the vibration is dominated by the Si-atoms.

Taraskin and Elliott [23] reported that the model constructed by using Van Beest potential[24] or Tsuneuki potential[25] were unable to reproduce the pronounced peak at $12.5 \mathrm{THz}\left(\approx 416.9 \mathrm{~cm}^{-1}\right)$. Similarly, phenomenological three-body atomic potential or semiphenomenological potentials were unsuccessful to reproduce the peak. The Feuston-Garofalini[26] potential did not have this deficiency but it was unable to reproduce other principal peaks of the VDOS. In fact, the simple Bethe lattice[27] and ab-inito MD-constructed model[28] were reported to reproduce all the principal peaks. Further, the occurrence of a high frequency doublet was a topic of debate but, Sarnthein et. al. [28] were able to demonstrate that the splitting is a general feature of any tetrahedral amorphous network rather than longitudinal-optic-transverse-optic (LO-TO) effect.

\subsection{The effective VDOS}

In their paper, Taraskin and Elliott.[29] gave a detailed analysis of the connection between the true vibrational density of states and the experimentally measured VDOS from an inelastic neutron experiment. They observed that only the effective VDOS can be measured with experimental techniques. The true VDOS and the effective VDOS are related via a correction function. Mathematically, the correction function is defined by[29],

$$
C(\omega) \simeq 1+\frac{A}{3} \sum_{\alpha} \frac{\overline{b_{\alpha}^{2}}}{m_{\alpha}}\left[\rho_{\alpha}\left(\omega_{j}\right)-\rho_{\alpha}^{(0)}\right]
$$

where, $\mathrm{A}=\frac{3<m>}{<\overline{b_{\alpha}^{2}} e^{-2 \bar{W}}>}$ is a constant depending upon the scattering length, the mass of the ions and the Debye-Waller factors. Similarly, the quantities 


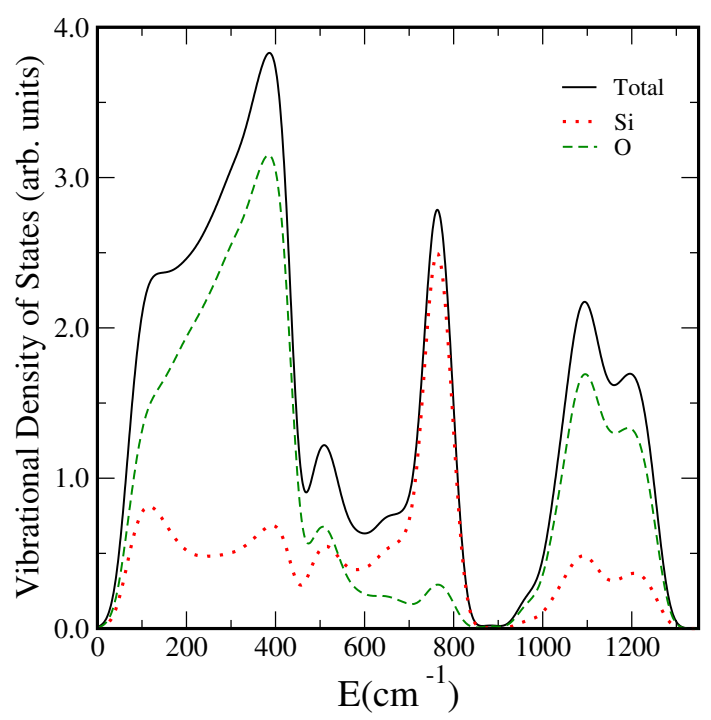

Figure 9: The obtained total VDOS (black,solid line), partial VDOS for $\mathrm{Si}$ (red, dotted line) and partial VDOS for O (green, dashed line).

$\rho_{\alpha}\left(\omega_{j}\right)=\frac{g_{\alpha}\left(\omega_{j}\right)}{g\left(\omega_{j}\right)}, \rho_{\alpha}^{(0)}=\frac{N_{\alpha} m_{\alpha}}{N<m>}$, represent the relative partial VDOS and the zero-frequency partial VDOS respectively for the species $\alpha$.

The true VDOS is connected to the effective VDOS by the relation[29],

$$
g_{\text {eff }}(\omega)=C(\omega) g(\omega)
$$

So with appropriate values of the constants and the relative partial VDOS, we evaluated the correction function and applied it to obtain the effective VDOS, which can be measured in an inelastic neutron experiment. The maximum and minimum values for the correction function were obtained as, $C_{\max }=1.495(1.48), C_{\text {min }}=$ $0.435(0.58)$ which were very close to the prescribed values (in brackets). Also, the normalization constant (G) for $\mathrm{SiO}_{2}$ was obtained as 1.10 which is very close to the suggested value of (1.14). The obtained effective VDOS was plotted with a direct comparison with the reduced neutron experiments [3],[5]. The effective VDOS is shown in Fig. 10. The plot of the effective VDOS shows some slight variation from the uncorrected original (true VDOS). Changes are observed at the peaks $390 \mathrm{~cm}^{-1}$ (increased effective density), $766 \mathrm{~cm}^{-1}$ (decreased effective density) and at the high frequencies $1095 \mathrm{~cm}^{-1}$ (increased effective density) and $1198 \mathrm{~cm}^{-1}$ (increased effective density).

\footnotetext{
${ }^{3} \boldsymbol{G}^{\mathbf{1}}$ : one-phonon reduced neutron scattering spectrum see(fig.5a,
}

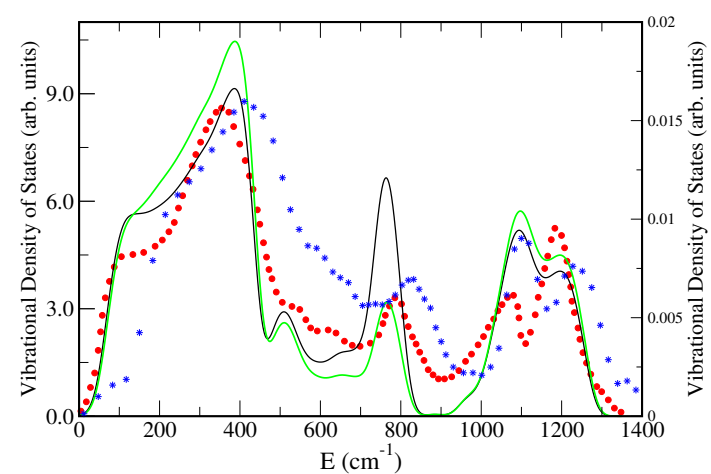

Figure 10: The obtained total VDOS as shown in Fig. 9 (black,solid line), effective VDOS obtained by Eq.(11) (green, solid line), experimental data (red ,dots)[3] ${ }^{3}$ and experimental data (blue, stars) [5]

We observe from Fig.10 that the two experiments don't exactly coincide. This makes the interpretation of our computed VDOS more challenging. It is self evident from the above figure that we have a general satisfactory agreement with the experiments regarding the occurrence of the major peaks in VDOS, while there are some discrepancies in weighting, between the experiment and theory.

\subsection{Vibrational localization}

Amorphous solids may possess localized vibrational modes. We can evaluate the localization different vibrational modes near the band edges oscillating with different frequencies by again using the inverse participation ratio (IPR) much like the case for electrons. The IPR can be easily calculated from the normalized displacement vectors, and the IPR for the vibrations can be defined (for mode $j$ ) as:

$$
\mathcal{I}=\frac{\sum_{i=1}^{N}\left|u_{i}^{j}\right|^{4}}{\left(\sum_{i=1}^{N}\left|u_{i}^{j}\right|^{2}\right)^{2}}
$$

The inverse participation ratio of a localized displacement is $\mathcal{I} \approx 1$ and the extended or de-localized displacement is close to $\mathcal{I} \approx 0$. A plot of vibrational IPR is shown in Fig.11. It can be seen that the low frequency band of the vibrations is de-localized with the only localized modes occurring at the frequency $\sim 31 \mathrm{~cm}^{-1}$. There is clear band gap $\left(\approx 135 \mathrm{~cm}^{-1}\right.$ or $\left.4.04 \mathrm{THz}\right)$ excepting a heavily localized state at $\sim 885 \mathrm{~cm}^{-1}$. As expected, these localized modes involve the motion of few atoms as opposed to the motion of nearly all the atoms in the de-localized frequencies.

The middle and bottom panels of Fig. 11 show, the partial or species projected vibrational localization of 


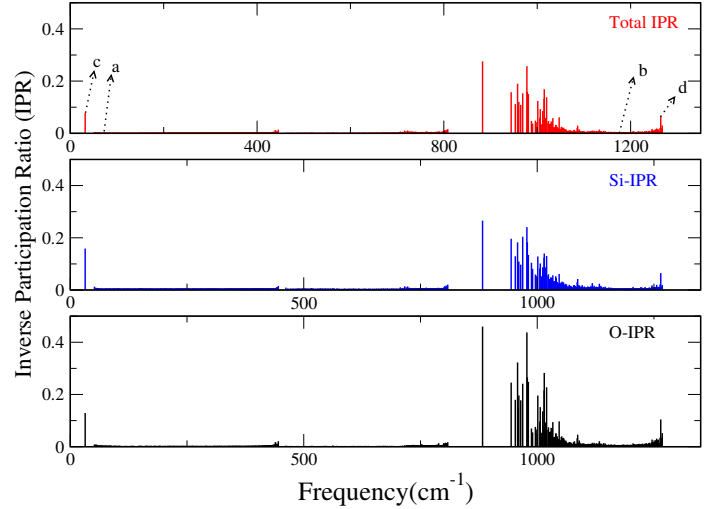

Figure 11: Plot of vibrational IPR: total IPR (top), Si-IPR (middle) and O-IPR (bottom)

the two atoms. It can be asserted that the contribution of oxygen atoms is more pronounced with respect to both vibration and localization. To obtain the speciesprojected IPR we use the same Equation (12) but the summation is restricted to particular atoms, in our case silicon or oxygen.

The modes were further analyzed by using visualization techniques 45 . The modes were investigated using an earlier recipe [31].

In this recipe, each atom is represented with a color code on the basis of the percentage of vibration it contributes to a particular frequency mode. The assignment is done in such a way that it includes at least 80 percent of the total vibrations in a particular mode.

In the above two Figs. 12.(a) and 12.(b), we analyze two extended modes with comparable values of IPR. In Fig 12.(a) the vibrations occurring at one of the low frequency mode $\left(72.38 \mathrm{~cm}^{-1}\right)$ is shown while Fig 12.(b) gives a visual description of a high frequency mode $\left(1176.38 \mathrm{~cm}^{-1}\right)$. The two plots resemble each other in most aspect, with the notable difference is that vibrations of silicon is more in low frequency mode (Fig. 12.(a)) than the high frequency mode (Fig. 12.(b)).

We also used this visualization technique to observe some of the prominent localized states, notably occurring at the frequencies $31.85 \mathrm{~cm}^{-1}$ and $1264.56 \mathrm{~cm}^{-1}$. The localized modes exhibit few atoms vibrating in the network, with some of the atoms contributing more than the 50 percent of the vibration at a particular frequency. The localized modes occurring well inside the optical band or the high frequency region of the spectrum were throughly studied, with the intent of identifying the pos-

\footnotetext{
${ }^{4}$ Jmol: an open-source Java viewer for chemical structures in 3D.

${ }^{5}$ VMD: a molecular visualization program [30].
}

Fig.12. (a) $72.38 \mathrm{~cm}^{-1}$ and IPR $=0.0025$

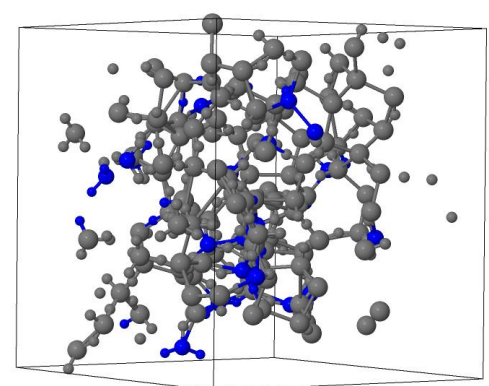

Figure 12: Visualization of an extended vibrational mode. Each atom shown is labeled with different colors with respect to the fraction of total vibration: grey $(>1 / 1024$ and $<1 / 256)$, blue $(>1 / 256$ and $<1 / 64)$, red $(>1 / 64$ and $<1 / 16)$, yellow $(>1 / 16$ and $<1 / 4)$ and green $(>1 / 4$ and $<1)$. The larger atom represents silicon $(\mathrm{Si})$ and the smaller one denote the oxygen $(\mathrm{O})$ atoms. The labels $(\mathrm{a}, \mathrm{b}, \mathrm{c}, \mathrm{d})$ on top of each figures are shown in Fig 11.(top panel)

Fig.12. (b) $1176.38 \mathrm{~cm}^{-1}$ and IPR $=0.0028$

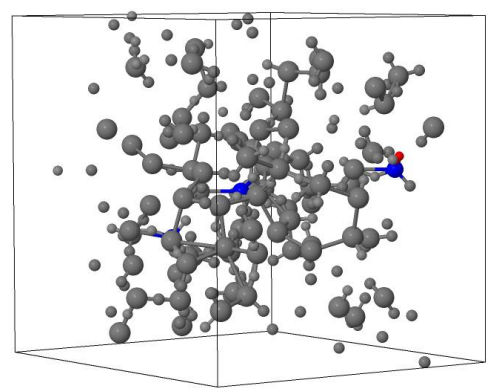

sible signatures of voids and to distinguish the possible pattern of vibrational localizations. These plots are shown in Fig. 12.(c) and Fig. 12.(d).

Fig.12. (c) $31.85 \mathrm{~cm}^{-1}$ and IPR $=0.0781$

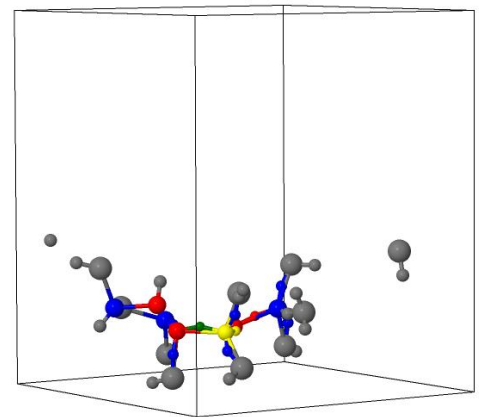

The above Fig. 12.(c) and Fig. 12.(d) depicts the two localized states, one occurring at the low frequency end 
and the other at the high frequency part of the spectrum. The comparison of the two localized modes shows that the vibrations are not as evenly distributed as in Fig. 12.(a) and Fig.12. (b), were most of the atoms had almost similar vibrational contribution. The localized state at the band gap at that frequency $\left(882.94 \mathrm{~cm}^{-1}\right)$ (not shown) is particularly peculiar as one of the oxygen atom has more than half of the total vibrational weight.

Fig.12. (d) $1264.56 \mathrm{~cm}^{-1}$ and IPR $=0.0653$

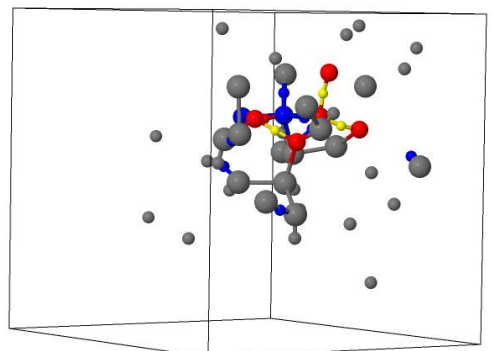

We also used the visualization techniques to obtain snapshots of the vibrational motions at a particular frequency. These several frames of snapshots were further visualized in VMD and were studied as movies. We found that these states to be consistent with the bending and stretching motions assigned earlier.

\subsection{Phase quotient}

The eigenvectors of the dynamical matrix have complete information about the atomic motions within the harmonic approximation. We can calculate the phase relationship among the neighbors of an atom by classifying whether the motion of atoms are in phase or out of phase with each other. The (Bell and Hibbins-Butler) [32] definition of phase quotient can be stated as [23],

$$
q_{j}=\frac{1}{N_{b}} \sum_{i} \sum_{i^{\prime}} \frac{u_{i}^{j} \cdot u_{i^{\prime}}^{j}}{\left|u_{i}^{j}\right| \cdot\left|u_{i^{\prime}}^{j}\right|}
$$

where, $N_{b}$ is the number of valance bonds, $u_{i}^{j}$ and $u_{i^{\prime}}^{j}$ are the normalized displacement vectors as defined earlier (section 5.4). In equation (13), $i$ sums over all the silicon atoms and $i^{\prime}$ enumerates all the oxygen atoms which are the neighbors of the silicon atom. A complete acoustic motion will have a value of 1 , while the optic like modes will attain a negative value for the phase quotient.

In Fig. 13 we show the plot of phase quotient $\left(q_{j}\right)$. The plot shows three clear branches with an abrupt change of phase quotient at around $\sim 450 \mathrm{~cm}^{-1}$ and the other jump at the band gap $\left(\sim 850 \mathrm{~cm}^{-1}\right)$, which indicates a different nature of optic vibrations[23],[33].

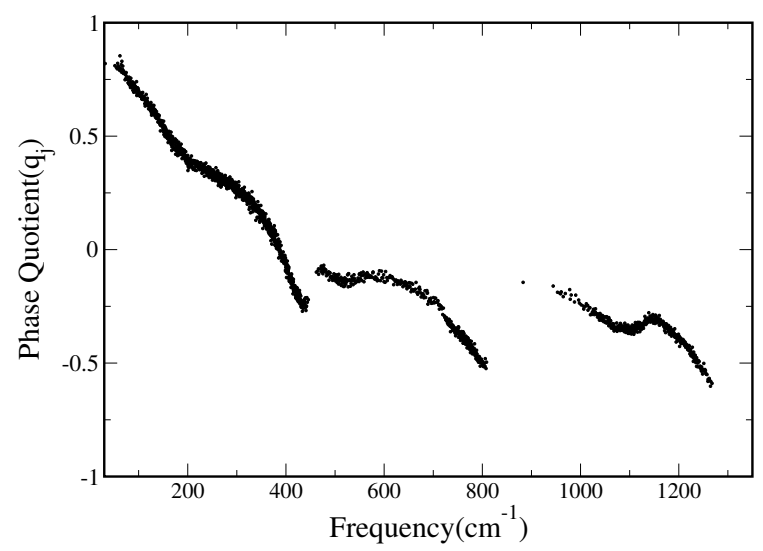

Figure 13: Phase Quotient $\left(q_{j}\right)$ versus the frequency.

\subsection{Stretching-Bending character}

To further investigate the modes, we studied the stretching character of our 648-atom model. The stretching and bending character defines the extent to which a mode is stretching type or bond-bending type and it can be defined as [33],

$$
S\left(\omega_{p}\right)=\frac{\sum_{m}\left|\left(u_{p}^{i}-u_{p}^{j}\right) \cdot \hat{r_{i j}}\right|}{\sum_{m}\left|u_{p}^{i}-u_{p}^{j}\right|}
$$

where, $u_{p}^{i}$ and $u_{p}^{j}$ are the eigen vectors of the $p^{t h}$ mode, $\hat{r_{i j}}$ is the unit vector parallel to the $m^{\text {th }}$ bond. $S(\omega)$ is close to unity when the mode of vibration is predominantly of bond-stretching type and will be close to 0 if the mode is of a bond-bending type. The plot of $S(\omega)$ is shown in Fig. 14.

The plot of $S(\omega)$ can be divided in three distinct regions similar to the phase quotient. The three regions can be distinguished with a clear gap at $\sim 450 \mathrm{~cm}^{-1}$ and $\sim 850 \mathrm{~cm}^{-1}$. The modes less than $\sim 450 \mathrm{~cm}^{-1}$ shows bond-bending type of character, the regions $\sim 450-850$ $\mathrm{cm}^{-1}$ shows a mixed type of behavior and the high frequency range shows a clear stretching character. The obtained $S(\omega)$ is in a good agreement with the previous calculations [33] with a slight difference in the amplitude.

To aid in the interpretation of these modes, we have included animations of several key modes as Supplementary Material associated with this paper. 


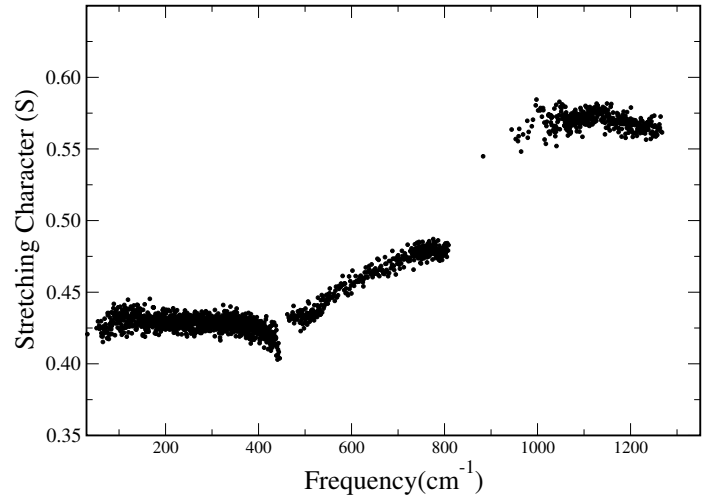

Figure 14: Stretching character $(S(\omega))$ versus the frequency $(\omega)$. The small values of $(\mathrm{S})$ is bond-bending, large values corresponds to optical streching modes.

\subsection{Phonon specific heat in the harmonic approxima- tion}

The evaluation of the specific heat within the harmonic approximation is straightforward with the help of the density of states, $g(\omega)$. The only significant provison is that not all of the vibrational excitations can be represented with a finite size model: vibrational excitations with wavelength larger than our supercell size are not included in the obtained VDOS data [34]. With the VDOS in hand, we may compute the $C_{v}(T)$ by the relation[35][36],

$$
C(T)=3 R \int_{0}^{E_{\max }}\left(\frac{E}{k_{B} T}\right)^{2} \frac{e^{E / k_{B} T}}{\left(e^{E / k_{B} T}-1\right)^{2}} g(E) d E
$$

where the VDOS, $g(E)$ is normalized to unity ${ }^{6}$. Further at low temperatures, the specific heat follows the Debye law so we calculated the term $C_{V}(T) / T^{3}$ and plotted it as follows,

In Fig. 15 we can see that the obtained plot of $C_{V}(T) / T^{3}$ with our 648-atom $\mathrm{SiO}_{2}$ model is in reasonable agreement with the experimental findings. It is to be noted that our peak is little shifted to the right and has somewhat reduced amplitude. But, our results are substantially improved over the previous simulation results of Taraskin and Elliott[23] using the van Beest potential [24]. A similar calculation was done by Horbach et. al[37] using 8016 ions with a larger box size but the results do not reproduce the $C_{V}(T) / T^{3}$ curve.

The authors[23],[37] have assigned this discrepancy in the plot of $C_{V}(T) / T^{3}$ to finite size effects and the

\footnotetext{
${ }^{6}$ see Ref.[36] Sec III, page 45
}

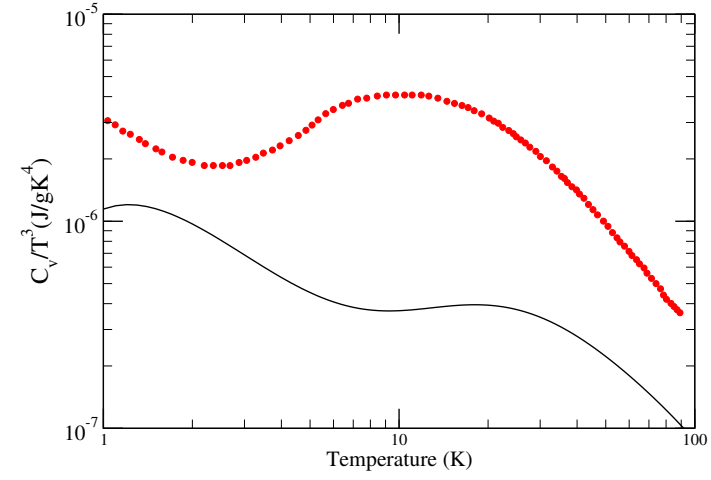

Figure 15: Low temperature dependence $C(T) / T^{3}$ : our model (black, solid line), Experiment. (Red, dotted line)[23].

cooling rates of their samples[37]. But, our calculation, with the same number of atoms (648) as Taraskin and Elliott and produced a better result. The calculation of Horbach et. al with a larger cell does not improve the results significantly. Thus, we can infer that the discrepancy between the theory and the experimental calculation may is due to inter-atomic interactions. Somewhat surprisingly, an $a b$ initio potential is required to qualitatively reproduce $C_{V}(T)$ for small $\mathrm{T}$.

The $C_{V}(T) / T^{3}$ curve has a noticeable plateau at a temperature $\sim 20 K$. The acoustic modes in the vibrational density of states (VDOS) has a Debye $\left(\sim \omega^{2}\right)$ spectrum. In the Debye spectrum an additional peak seen in the region $30-120 \mathrm{~cm}^{-1}$, usually referred as "Boson peak". We calculated the specific heat by excluding the frequencies lying in the region of "Boson peak"and notable changes were observed at the plateau of the $C_{V}(T) / T^{3}$ graph around the region $(20 \mathrm{~K})$. We illustrate these modes in Fig. 16. A representative example in this frequency range is the extended transverse mode of Fig. 12.(a). It has been shown that the Boson peak satisfies the Ioffe-Regel criterion[38], which is the reason many authors have proposed this to be a hybridized state of the acoustic band and the optic band. In agreement with Taraskin and Elliott we note that these are transverse-bond bending rather than stretching excitations.

\section{Conclusions}

We found that our model of 648 -atom $\mathrm{SiO}_{2}$ reproduces most of the known experimental properties while improving on some earlier calculations. The structure of our 648-atom $\mathrm{SiO}_{2}$ model is in reasonable agreement with the experimental results as obtained from the experimental techniques of diffraction and inelastic neu- 


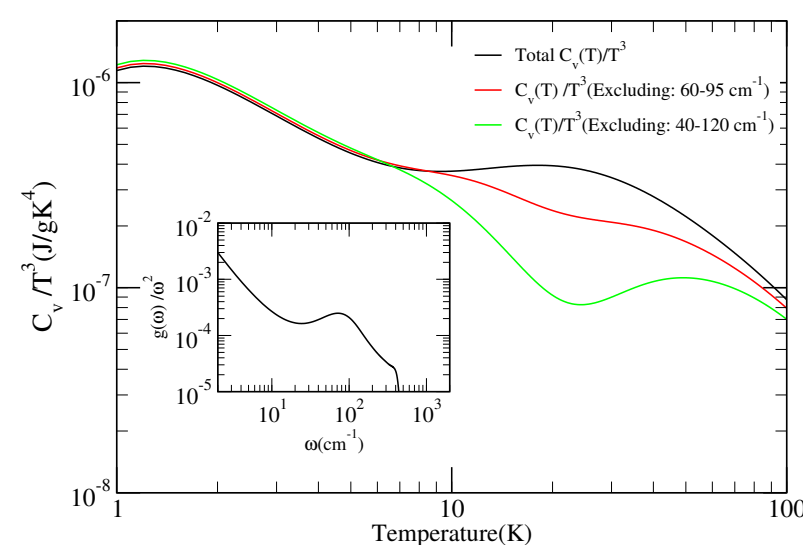

Figure 16: Low temperature dependence $C(T) / T^{3}$ : Black (total), Red (excluding the frequency range: $60-95 \mathrm{~cm}^{-1}$ ), Green (excluding the frequency range: $40-120 \mathrm{~cm}^{-1}$ ). INSET: Plot of $g(\omega) / \omega^{2}$ versus the frequency.

tron scattering, and is "fully first principles", perhaps the first such calculation, in the sense that the supercell geometry is optimized to minimize the total energy for the Hamiltonian we employ. The vibrational properties were analyzed with the help of eigenfrequencies and eigenvectors, obtained by diagonalizing the dynamical matrix. The VDOS obtained with our method showed a good agreement with the experimental data. It was able to produce all the principal peaks obtained in the experimental measurements on amorphous $\mathrm{SiO}_{2}$. In addition to the principal peaks, there was a peak observed at the frequency $\sim 495-507 \mathrm{~cm}^{-1}$ in both the 192-atom[1] and the current 648-atom model.

The localization of vibrational modes was also studied through the evaluation of inverse participation ratio (IPR) and with the help of the visualization techniques. We found that these modes were consistent with the different types of motion (rocking, bending, etc.) previously assigned by different authors [22], [23]. A through analysis of the localization was done by using visualization techniques including animations in the Supplementary Material. In addition, we were able to make an improved prediction for the low temperature specific heat compared to previous work [23][37]. We were also able to verify that plateau observed $(\sim 20 \mathrm{~K})$ in the plot of specific heat $\left(C(T) / T^{3}\right)$ was due to the "Boson peak"seen in the VDOS. We provide some evidence that the modes associated with Boson peak may be computed and analyzed in simulation.

In conclusion, we found that our 648-atom model improved the understanding of a-SiO 2 while describing the structural, electronic and vibrational properties and clarifying the importance of interatomic potential and model size on vibrational quantities.

\section{Acknowledgment}

The authors acknowledge the NSF under grant number DMR 1507670. We thank the Ohio Supercomputer Center for providing computing resources.

\section{Supplementary Material}

To further aid in the interpretation of the modes and as a pedagogic tool, we animated certain modes of interest - indicating the motion of the cell in specific pure modes (with the amplitude exaggerated, of course). As assortment of localized and extended modes are given. We choose these specific modes for the animation because they have some unique characteristics about their motion. These modes have been properly labeled in Fig. 17. The phase quotient and stretching characters of these modes may be see in Fig. 13 and Fig. 14.

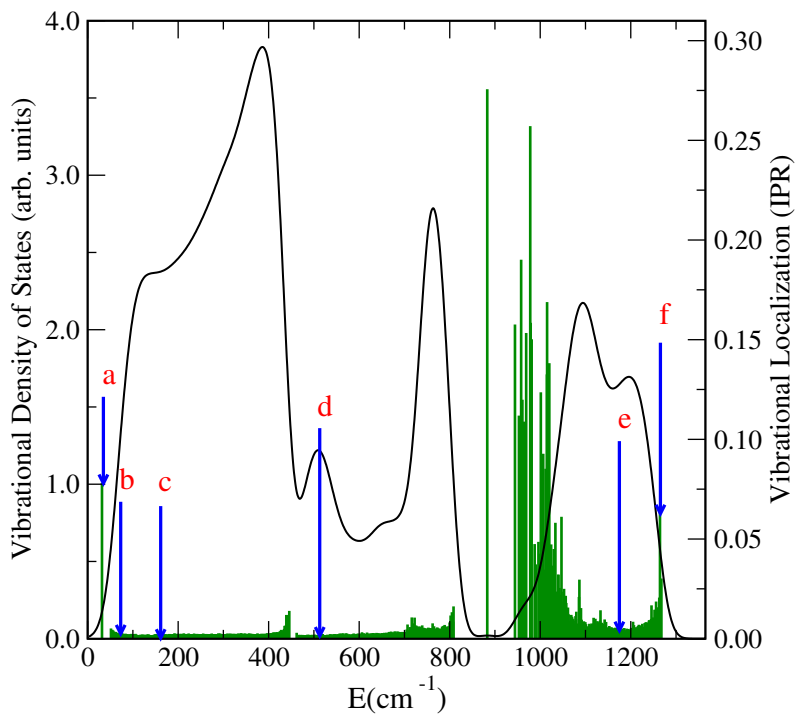

Figure 17: The Vibrational Density of States (black,solid line), the IPR (green,drop lines).

- (a) $31 \mathrm{~cm}^{-1}$, represents the first localized mode in a IPR-plot (Fig. 11), there is a unique torsional motion involving motion of only few atoms.

- (b) $72 \mathrm{~cm}^{-1}$, depicts the well know Boson-peak region of the VDOS (see Fig.16), it occurs in a region of well extended mode of vibrational motion and one can see that almost all atoms are in motion at this mode. 
- (c) $160 \mathrm{~cm}^{-1}$, it represents another well extended region of vibrational motion.

- (d) $514 \mathrm{~cm}^{-1}$, this represents the region of small peak in the VDOS at the frequency $(500-520 \mathrm{~cm}-$ $1)$. This peak was also signatures of some of earlier calculations[1].

- (e) $1176 \mathrm{~cm}^{-1}$, we in particular choose this frequency to compare a extended mode occurring well inside the optical region of vibration with the modes occurring in acoustic region of vibration. It is evident from Fig. 12 (a) and Fig. 12 (b) that both of these modes have a similar values of IPR and the vibrations occurring in different atoms are well distributed.

- (f) $1264 \mathrm{~cm}^{-1}$, as above, we considered this frequency to compare two localized modes occurring in the two different regions (acoustic and optical) of vibrations. one can see that very few atoms take part in the motion and the stretching motion dominates in optical region.

Note that the usual picture of a specific molecular mode at given frequency may be qualitatively correct, but the true eigenstates usually exhibit non-locality and often mode mixing.

\section{References}

[1] D. Tafen, D. A. Drabold, Phys.Rev.B 71 (2005) 054206.

[2] J. M. Soler, E. Artacho, J. D. Gale, A. Garca, J. Junquera, P. Ordejn, D. Snchez-Portal, Journal of Physics: Condensed Matter 14 (2002) 11

[3] F. L. Galeener, A. J. Leadbetter, M. W. Stringfellow, Phys.Rev.B 27 (1983) 1052

[4] D. Price, J. Carpenter, Journal of Non-Crystalline Solids 92 (1987) 153.

[5] M. J. Carpenter, D. L. Price, Phys.Rev.Lett 54 (1985) 441.

[6] F. L. Galeener, G. Lucovksy, Phys.Rev.Lett 37 (1976) 1474.

[7] C. T. Kirk, Phys.Rev.B 38 (1988) 1255.

[8] D. A. Drabold, Eur.Phys.J. B 68 (2009) 1.

[9] D. Tafen, D. A. Drabold, Phys.Rev.B 68 (2003) 165208.

[10] A. Pasquarello, J. Sarnthein, R. Car, Phys.Rev.B 57 (1998) 22.

[11] W. Jin, P. Vashistha, R. Kalia, J. P. Rino, Phys.Rev.B 48 (1993) 9359

[12] X. Zhang, Theoretical studies of structural, electronic and optical properties of amorphous chalcogenide materials, Ph.d dissertation, Ohio University, Department of Physics (2001).

[13] D. A. Drabold, Molecular Dynamics Simulations of Network Glasses, Vol. 17, World Scientific, Singapore, 2000, p. 635, and O. F. Sankey (unpublished).

[14] P. Johnson, A. C. Wright, R. N. Sinclair, Journal of NonCrystalline Solids 58 (1983) 109.

[15] R. M. V. Ginhoven, H. Jonsson, L. R. Corrales, Phys.Rev.B 71 (2005) 024208

[16] J. Neuefeind, K.-D. Liss, Phys.Chem 100 (1996) 1341

[17] S. Roux, V. Petkov, J. Appl. Cryst. 43 (2010) 181.
[18] S. King, Nature 213 (1967) 1112.

[19] J. Rino, I. Ebbsjo, R. K. Kalia, A. Nakano, P. Vashishta, Phys.Rev.B 47 (1999) 3053.

[20] Y. Li, D. A. Drabold, IET Circuits, Devices and Systems 9 (2015) 13.

[21] M. Cobb, D. A. Drabold, R. L. Cappelletti, Phys.Rev.B 54 (1996) 12162

[22] R. J. Bell, P. Dean, Discuss. Faraday Soc. 50 (1970) 55.

[23] S. N. Taraskin, S. R. Elliott, Phys.Rev.B 56 (1997) 8605

[24] B. W. H. van Beest, G. J. Kramer, R. A. van Santen, Phys.Rev.Lett. 64 (1990) 1955.

[25] S. Tsuneyuki, M. Tsukada, H. Aoki, Y. Matsui, Phys.Rev.Lett. 61 (1988) 869

[26] S. Garofalini, J. Non-Cryst. Solids 120 (1990) 1

[27] R. Laughlin, J. Joannopoulos, Phys.Rev.B 16 (1977) 2942

[28] J. Sarnthein, A. Pasquarello, R. Car, Science 275 (1997) 1925.

[29] S. N. Taraskin, S. R. Elliott, Phys.Rev.B 55 (1997) 117.

[30] A. D. W. Humphrey, K. Schulten, J. Molec. Graphics 14 (1996) 33.

[31] S. M. Nakhmanson, D. A. Drabold, Phys.Rev.B 58 (1998) 15325

[32] R. J. Bell, D. Hibbins-Butler, J.Phys.C 8 (1975) 787.

[33] N. Zotov, I. Ebbsjo, D. Timpel, H. Keppler, Phys.Rev.B 60 (1999) 6383.

[34] D. A. Drabold, Molecular Dynamics Simulations of Network Glasses, Vol. 17, World Scientific, Singapore, 2000, p. 634.

[35] S. M. Nakhmanson, D. A. Drabold, Phys.Rev.B 61 (2000) 5376.

[36] A. Maradudin, E. Montroll, G. H. Weiss, Theory of lattice dynamics in the harmonic approximation, Academic Press, New York, 1963, p. 123

[37] J. Horbach, W. Kob, K. Binder, J.Phys.Chem.B 103 (1999) 4104.

[38] S. N. Taraskin, S. R. Elliott, Europhys.Lett 39 (1997) 37. 